\title{
Adverse Effects of the COVID-19 Vaccine Reported by Lecturers and Staff of Kabul University of Medical Sciences, Kabul, Afghanistan
}

\author{
Mahmoodullah Azimi $\mathbb{D}^{\prime}$ \\ Wazhma Masoom Dehzad (D) \\ Mohammad Asif Atiq (D) \\ Barin Bahain $\mathbb{D}^{\prime}$ \\ Abdullah Asady $\mathbb{D D}^{2}$ \\ 'Department of Clinical Pharmacology, \\ Kabul University of Medical Sciences, \\ Kabul, Afghanistan; ${ }^{2}$ Department of \\ Microbiology, Kabul University of Medical \\ Sciences, Kabul, Afghanistan
}

\begin{abstract}
Purpose: To evaluate the occurrence, extent, and severity of adverse reactions associated with the vaccine of COVID-19 (ChAdOx1 nCoV-19 vaccine or AstraZeneca) among Kabul University of Medical Sciences staff.

Patients and Methods: A retrospective observational, interview-based study was conducted from 4 to 20 April, 2021, to evaluate the adverse reactions associated with the vaccine of COVID-19 (ChAdOx1 nCoV-19 vaccine or AstraZeneca) among the staff and lecturers of the Kabul University of Medical Sciences, Kabul, Afghanistan. Participants were interviewed following the administration of the first dose of the AstraZeneca vaccines. They were asked to report any adverse reactions that occurred within 8-10 days after vaccination. The frequency, duration, severity, and outcome of the reactions were recorded. Association of the adverse reactions was analysed with the ages of participants and previous infection with SARS CoV-2.

Results: The most common adverse reactions reported by the participants were muscle pain $(68.3 \%)$, local pain $(58.8 \%)$ at the site of injection $(68.3 \%)$, fever $(66.3 \%)$ and fatigue $(66.3 \%)$. Almost half of the respondents reported chills, joint pain and headache after receiving the first shot of the vaccine. The frequency of adverse reactions was higher in participants aged 40 years or less, and in those previously infected with SARS CoV-2. The severity of most adverse reactions was mild to moderate. No serious case or death was reported.
\end{abstract}

Conclusion: The adverse reactions reported by the participants were mild to moderate in severity, and for a short duration. The findings of this study help us to address the vaccine hesitancy caused by worries about severe adverse effects associated with the COVID-19 vaccine.

Keywords: adverse effects, COVID-19, vaccine, ChAdOx1 nCoV-19, AstraZeneca

\section{Introduction}

Coronavirus Disease 2019 (COVID-19) caused by Severe Acute Respiratory Syndrome Coronavirus-2 (SARS CoV-2) is changing the pattern of emergency services around the world as the disease is causing a substantial public health emergency. The infection first appeared in Wuhan, China in December 2019 and spread rapidly throughout the world quickly. ${ }^{1}$ As of June 2021, the COVID-19 has been reported to infect more than 173 million people and took lives of more than 3.72 million people worldwide. ${ }^{2}$ To overcome this global crisis, the vaccination strategy against COVID-19 is considered as the final answer. ${ }^{3}$ The COVID-19
Correspondence: Abdullah Asady Department of Microbiology, Kabul University of Medical Sciences, Kabul, Afghanistan

$\mathrm{Tel}+93731087928$

Email asady_abdullah@yahoo.com 
vaccine triggers the immune system to be prepared to fight against the virus by producing antibodies. Most of the COVID-19 vaccines utilize $\mathrm{S}$ protein, a protein that supports the virus to enter the cells and start the infection process. ${ }^{4,5}$ Many vaccines have been introduced and at least 13 different vaccines (across 4 platforms) are currently in use. 6

These vaccines are either Messenger RNA (mRNA) vaccines such as Pfizer-BioNTech and the Moderna COVID-19 vaccines or Vector vaccines such as Janssen/ Johnson \& Johnson and AstraZeneca vaccines, Protein subunit vaccines, and inactivated vaccines such as Sinopharm vaccine. In the mRNA vaccines, a genetically engineered mRNA is used to deliver the information for $S$ protein, while DNA vector vaccine uses a modified version of a different harmless virus like chimpanzee adenovirus. The DNA vector is employed as a template to build new chimp adenovirus replicas and produce the viral protein that triggers an immunological response. The chimpanzee adenovirus is injected into humans and it hook to the host cells. ${ }^{7}$ DNA is released into the cytoplasm and then migrates to the nucleus of the cell. It is not incorporated into cellular DNA, but rather is turned into mRNA by host enzymes, which migrates back into the cytoplasm and interacts with host cell ribosomes to produce translated proteins. Following the production of copies of $S$ protein and their expression on the surface of host cells, the host immune system begins to recognize and react to viral particles. ${ }^{4,5,7,8}$ Subunit vaccines contain the harmless $\mathrm{S}$ protein only. ${ }^{4,5,8}$

The first mass vaccination program started in early December 2020 in Europe. ${ }^{9}$ Until June 2021, more than 1.9 billion doses of the COVID-19 vaccine have been administered globally. In Afghanistan, the COVID-19 vaccination was first administered on February 23, 2021. The ChAdOx1 nCoV-19 vaccine (AstraZeneca) was administered to healthcare workers in the first phase of the vaccination process. The safety profile for the AstraZeneca vaccine was proved by randomized controlled trials. ${ }^{10}$ To reach the national goal of herd immunity, it is essential to expand the inoculation rate of the COVID-19 vaccine.

Hesitancy and reluctance in taking the COVID-19 vaccines and apprehensions about them were present among the general population all along during the implementation of vaccination programs. Many factors, such as the speedy production of vaccines, lack of information, and uncertainty about effects emerging after vaccination as well as the news of side effects spreading through media, have given rise to suspicion and fear among Afghans. ${ }^{11,12}$

Studies have reported about adverse effects of the COVID-19 vaccines including some experiencing severe reactions, such as deep vein thrombosis, transverse myelitis, and even anaphylactic shock. ${ }^{10,13,14}$ Misinformation about the safety of the vaccine through media has caused significant anxiety and concerns among people. ${ }^{15}$ Furthermore, no large-scale study had been done to investigate the adverse effects of immunization against COVID19 in Afghanistan. We intended to evaluate the occurrence, extent, and severity of adverse reactions associated with the COVID-19 vaccine among the staff and lecturers of Kabul University of Medical Sciences (KUMS). The findings will provide baseline data and inform policymakers on the safety of the COVID-19 vaccine during the national vaccination program, which is currently being implemented.

\section{Methodology}

This is a retrospective observational study conducted from April 4 to April 20, 2021. The data collection method was census and all the vaccinated staff and lecturers of KUMS were included. A total of 400 lecturers and staff took part in the study. All participants had received the first dose of the COVID-19 vaccine (ChAdOx1 nCoV-19), manufactured by AstraZeneca. The lists and relevant information of the vaccinated staff were obtained from the vaccination team of the KUMS.

The study protocol was reviewed and approved by Research and Ethics committee of Department of Clinical Pharmacology, KUMS (Protocol no. 71). All participants were given a brief introduction about the purpose and outcome of the study and were asked to provide their informed consent prior to the study. All aspects of the study observe the ethical standards of the relevant institutional committees on studies involving human contacts and the Declaration of Helsinki released in 1975 and subsequent revisions. The participation was completely voluntary, and they could withdraw from the study anytime without any consequence. Once they agreed to participate in the survey, the participants were interviewed to report any adverse reaction they suffered within 8-10 days after receiving the first dose of their vaccines.

In order to ensure no adverse reaction was ignored, the participants first asked to report any reactions they might have suffered after receiving the vaccine, then they were asked about a list of adverse reactions if they have 
experienced. The list was provided using the CDC leaflet and reports published by randomized control trials on the COVID-19 vaccine. ${ }^{16}$

The data were analyzed using IBM SPSS statistics version 25. Descriptive statistics and bi-variable (Chi square) analyses were performed. The responses were presented as frequency and percentage. Associations between adverse effects of the COVID-19 vaccine reported by participants, participants' ages and previous infection with SARS CoV-2 were investigated using Chi square. A p-value of $<0.05$ considered statistically significant at $95 \%$ confidence interval.

\section{Results}

\section{Demographic Characteristics}

A total of 400 participants took part in the study. Table 1 shows the demographic characteristics, previous infection with SARS CoV-2 and the type of vaccine received by the participants. The mean age of the participants was 39 \pm 12.04 , with more than half of them being 40 years old or below. Majority $(81.3 \%)$ of the participants were males. Nearly one-third of them were doctors, and the others were either administrative or supporting staff. Almost $60 \%$ of the study population reported to be infected by the SARS

Table I Demographic Characteristics, Previous Infection with SARS CoV-2 and Type of Vaccine Received by the Participants $(\mathrm{N}=400)$

\begin{tabular}{|l|l|}
\hline Characteristics & Frequency (n and \%) \\
\hline $\begin{array}{c}\text { Sex } \\
\text { Male }\end{array}$ & $325(81.3)$ \\
Female & $75(18.8)$ \\
\hline Age (Mean \pm SD) & $39.6 \pm 12.04$ \\
\hline $\begin{array}{l}\text { Age groups } \\
\leq 40 \text { years }\end{array}$ & $227(56.8)$ \\
$>40$ years & $173(43.3)$ \\
\hline $\begin{array}{l}\text { Employment } \\
\text { Doctor } \\
\text { Administration officer } \\
\text { Support staff }\end{array}$ & $122(30.5)$ \\
\hline Previous infection with SARS CoV-2 & $173(43.3)$ \\
Yes & $105(26.3)$ \\
\hline No & $238(59.5)$ \\
\hline $\begin{array}{l}\text { Type of COVID-19 vaccine } \\
\text { ChAdOxI nCoV-19 vaccine (AstraZeneca) }\end{array}$ & $400(100)$ \\
\hline
\end{tabular}

CoV-2 previously. All participants received the first dose of ChAdOx1 nCoV-19 vaccine (AstraZeneca).

\section{Frequency of Adverse Reactions}

Figure 1 shows the frequency of adverse reactions reported by the study participants within $8-10$ days after receiving the first dose of vaccine. A total of 374 (93.5\%) respondents reported at least one adverse reaction following vaccination. The most common systemic adverse reaction reported by the participants was muscle pain (68.3\%) followed by fever $(66.3 \%)$ and fatigue $(66.3 \%)$. Almost half of the respondents reported chills, joint pain and headache after receiving the first shot of the vaccine. Less common systemic adverse reactions reported were sore throat $(26.8 \%)$, nausea $(13 \%)$, and diarrhea (11.8\%). Insomnia (reported by nine participants), anorexia (reported by six), drowsiness (reported by three) and impotence (reported by two) were rare systemic adverse effects. The most common local adverse reaction reported by the participants was local pain $(58.8 \%)$ followed by swelling (15.3\%). Redness at the site of injection (reported by four participants), stiffness (reported by two) and itching (reported by only one) were rare adverse reactions.

More than half (56.1\%) of the adverse reactions lasted for one day or less, whereas $23.1 \%$ lasted two days, $12 \%$ lasted three days, $2.5 \%$ lasted four days and only one case lasted for ten days. All reported adverse reactions were self-limiting without any specialized care except for one participant who needed hospital admission and oxygen therapy for one night. No death was reported following vaccination among the study participants.

Table 2 shows the reported adverse effects of the COVID19 vaccine and their association with the ages of the participants. Overall, the prevalence of adverse reactions was slightly higher in participants aged $\leq 40$ years as compared to those aged $>40$ years $(94.3 \%$ versus $92.5 \%$ respectively). However, this difference was not found to be statistically significant. Reports of fever were significantly higher among participants $\leq 40$ years than $>40$ years old $(70.5 \%$ and $60.7 \%$, respectively; $\mathrm{p}=0.04)$. Likewise, muscle pain was more frequent among those aged $\leq 40$ years as compared to those aged $>40$ years ( $73.6 \%$ and $61.3 \%$, respectively; $\mathrm{p}=0.009$ ).

Table 3 shows the reported adverse reactions following COVID-19 vaccine and their association with previous infection of SARS CoV-2. Adverse reactions due to COVID-19 vaccine were more common among participants who were previously infected with SARS CoV-2 compared to the opposite group (96.2\% and 89.5\%, 


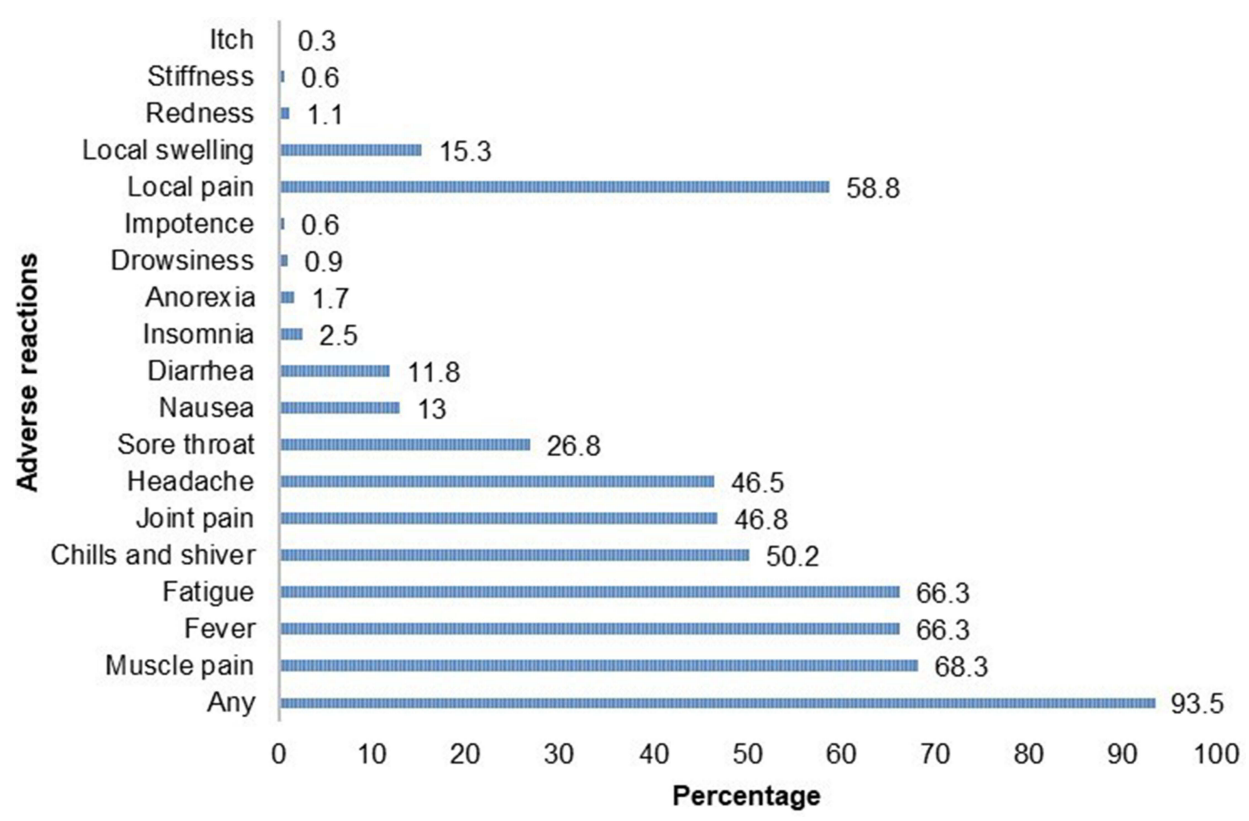

Figure I Adverse reactions reported by the participants after receiving the first dose of the COVID-19 vaccine (in percentage).

respectively; $\mathrm{p}=0.008$ ). Chi-square analysis also revealed that fatigue was significantly higher in persons previously infected with SARS CoV-2 compared to those not infected (70.6\% and 59.9\%, respectively; $\mathrm{p}=0.026)$.

\section{Discussion}

Since the beginning of vaccine production, people have raised their concerns and worries over adverse events and

Table 2 Adverse Reactions Reported by the Participants and Their Association with Participants' Ages

\begin{tabular}{|l|l|l|l|}
\hline \multirow{2}{*}{} & \multicolumn{3}{|l|}{ Frequency (n and \%) } \\
\cline { 2 - 4 } & $\begin{array}{l}\text { Age } \leq \mathbf{4 0} \\
\text { Years }\end{array}$ & $\begin{array}{l}\text { Age > 40 } \\
\text { Years }\end{array}$ & $\begin{array}{l}\text { Chi- } \\
\text { Square }\end{array}$ \\
\cline { 2 - 4 } & $\mathbf{( n = 2 2 7 )}$ & $\mathbf{( n = 1 7 3 )}$ & p value \\
\hline Adverse reactions & $214(94.3)$ & $160(92.5)$ & 0.472 \\
(any) & & & \\
Headache & $111(48.9)$ & $75(43.4)$ & 0.271 \\
Fatigue & $157(69.2)$ & $108(62.4)$ & 0.158 \\
Chills and shiver & $120(52.9)$ & $81(46.8)$ & 0.231 \\
Diarrhea & $27(11.9)$ & $20(11.6)$ & 0.918 \\
Fever & $160(70.5)$ & $105(60.7)$ & 0.04 \\
Joint pain & $110(48.5)$ & $77(44.5)$ & 0.433 \\
Muscle pain & $167(73.6)$ & $106(61.3)$ & 0.009 \\
Nausea & $28(12.3)$ & $24(13.9)$ & 0.650 \\
Sore throat & $61(26.9)$ & $46(26.6)$ & 0.950 \\
Local pain & $136(59.9)$ & $99(57.2)$ & 0.589 \\
Local swelling & $37(16.3)$ & $24(13.9)$ & 0.504 \\
\hline
\end{tabular}

risks associated to COVID-19 vaccine. Factors such as knowledge about vaccines, possible risks, personal experiences, religious or cultural beliefs, and political motives as well as social and economic status determine the level of public trust on vaccines. ${ }^{17}$ Some adverse reactions may not have been reported in pre-clinical trials due to their lower frequency, smaller number of people participating in trials and other similar restrictions. Thus, post-vaccination monitoring of the adverse reactions is important to inform the

Table 3 Adverse Reactions Reported by the Participants and Their Association with Previous SARS CoV-2 Infection

\begin{tabular}{|l|l|l|l|}
\hline \multirow{2}{*}{} & \multicolumn{3}{|l|}{ Frequency (n and \%) } \\
\cline { 2 - 4 } & \multicolumn{2}{|l|}{ Previous Infection with SARS CoV-2 } \\
\cline { 2 - 4 } & Yes & No & Chi-Square \\
\cline { 2 - 4 } & $\mathbf{( n = 2 3 8 )}$ & $\mathbf{( n = 1 6 2 )}$ & P value \\
\hline Adverse reactions (any) & $229(96.2)$ & $145(89.5)$ & 0.008 \\
Headache & $112(47.1)$ & $74(45.7)$ & 0.786 \\
Fatigue & $168(70.6)$ & $97(59.9)$ & 0.026 \\
Chills and shiver & $123(51.7)$ & $78(48.1)$ & 0.488 \\
Diarrhea & $29(12.2)$ & $18(11.1)$ & 0.743 \\
Fever & $166(69.7)$ & $99(61.1)$ & 0.073 \\
Joint pain & $113(47.5)$ & $74(45.7)$ & 0.723 \\
Muscle pain & $167(70.2)$ & $106(65.4)$ & 0.318 \\
Nausea & $33(13.9)$ & $19(11.7)$ & 0.533 \\
Sore throat & $70(29.4)$ & $37(22.8)$ & 0.145 \\
Local pain & $143(60.1)$ & $92(56.8)$ & 0.511 \\
Local swelling & $36(15.1)$ & $25(15.4)$ & 0.933 \\
\hline
\end{tabular}


public and policymakers of the safety and possible severe reactions of the vaccine.

This study reports the adverse reactions reported by the participants after receiving the first dose of AstraZeneca vaccine (ChAdOx1 nCoV-19). The most common adverse reactions reported were muscle pain, fever, fatigue, and local pain at the site of injection. Almost half of the respondents reported chills, joint pain and headache after receiving the first shot of the vaccine. Less frequently reported systemic adverse reactions were sore throat, nausea, diarrhea and swelling at the site of injection. Rare adverse reactions were insomnia, anorexia, drowsiness and impotence. The severity of most adverse reactions was mild to moderate. No serious case requiring hospitalization was reported except in an elderly female medical staff, who needed overnight hospitalization as her blood oxygen saturation started to fall but her condition began to improve in 48 hours after vaccination. Overall, the side effects had improved within a few days.

Our findings conform to the interim analysis of four clinical trials on the ChAdOx1 nCoV-19 vaccine, which reported that tenderness at the site of injection (63.7\%), pain at the site of injection (54.2\%), headache (52.6\%), and fatigue $(53.1 \%)$ were most common adverse effects. The severity of the reported adverse effects was mild to moderate in the majority of cases after vaccination, and all symptoms usually disappeared within a few days of vaccination. ${ }^{3,10}$ Moreover, the findings are also consistent with the adverse reactions reported after receiving the ChAdOx1 nCoV-19 vaccine by healthcare workers in Nepal. ${ }^{18}$

Compared to these clinical trial reports, our findings indicate much higher local and systemic adverse effects after vaccination. These differences might be due to ethnicity difference, including previously not infected with COVID-19 and more aged participants. In the previous clinical trials, the proportions of South Asian participants were very low. In our study, none of the participants was aged 65 or older, while in a safety analysis of the clinical trials, it was $8.9 \%$. The underestimation of incidence and severity of adverse effects might be due to participating of more elderly individuals in clinical trials, as these studies generally reported mild reactions and less adverse effects in older adults $\left(\geq 65\right.$ years old). ${ }^{3}$

Of the 6 vaccinated individuals who presented to the hospital with side effects, 2 of them had blood clots, one with AstraZeneca and the other with Pfizer. ${ }^{11,17}$ Taking into account the facts and figures of worldwide studies, the findings in our study are precisely reporting the common side effects with ChAdOxl n COVID-19 only.

There was no incidence of anaphylaxis among respondents and only four participants reported mild acute hypersensitivity reactions, which were self-limiting. Huh et al reported that the incidences of anaphylactic shock associated with vaccination tended to rise in Korea. There were 96 suspected cases of anaphylaxis $(96 / 771,284,0.01 \%)$ after vaccination in Korea as of 26/3/2021. ${ }^{19}$

Afghanistan has not been an exception since the only vaccine available was ChAdOxl n COVID-19, the most controversial of them all. The reports of thrombocytopenia and blood clots associated with this vaccine made it even harder to convince people to participate in taking the vaccine. ${ }^{16,19}$ As the efficacy and safety of vaccines were approved subsequently, compared and their postvaccination side effects were observed. It was found that ChAdOxl n COVID-19 had a higher incidence of side effects; however, most of these were local and selflimiting rarely causing hospitalization and none was lifethreatening. ${ }^{11,16}$

In studies conducted to compare the side effects of three major vaccines, Sinopharm, BioN tech, and AstraZeneca, all three vaccines were associated with similar side effects (fatigue, injection site pain and swelling, headache, sleepiness and laziness, chills, myalgia, joint pain, and fever), though the severity and number of adverse reactions differed with the type of vaccine. ${ }^{3,10,11}$ Injection site pain and swelling, chills, sleepiness, and laziness were more common with AstraZeneca compared to the other two vaccines. ${ }^{11,12,17,20}$

Adverse reactions due to COVID-19 vaccine were more common among participants who were previously infected with SARS CoV-2 compared to the participants previously not infected. Several studies have also reported a higher rate of adverse reactions among participants previously infected with COVID-19 compared to those not infected. ${ }^{11,12,21}$

\section{Strength and Limitations}

The main strength of our study was the little chance of non-response bias, it was an interview-based study, and where all vaccinated population of KUMS staff took part. However, it was conducted only after the first dose administered, which limits the information about the intensity and severity of side effects reported after the second dose of vaccine. As other studies show, the number of side effects was higher after the first dose and more intense 
after the second dose of the COVID-19 vaccine. This information after the second dose is yet to be explored in the population under study. Smaller sample size may also make it difficult to generalize the findings; however, due to lower coverage and shortage of vaccines in Afghanistan, the findings of this study could provide a useful insight into the situation and may play an important role in reducing vaccine hesitancy among public.

\section{Conclusion}

The short-term adverse reactions of the vaccine were mild to moderate in severity and short in duration. Most of the adverse effects were tolerable and similar to the reports of manufacturing company. The findings of this study help to address the vaccine hesitancy caused by worries about severe adverse effects associated with the COVID-19 vaccine. However, it is early to proclaim that vaccination is entirely safe and further studies are required to observe the long-term side effects as well as to determine the efficacy of full vaccination and immune response. In the current situation when we are struggling to contain and overcome the pandemic that has proven to be deadly and is crippling the health systems around the world, the benefits of vaccination outweigh the risk of side effects after vaccination. Moreover, our study in Kabul included healthcare workers such as doctors, administration, and supporting staff of KUMS who did not show resistance to vaccination. However, to convince the general population in Afghanistan, proper awareness programs and timely sharing of knowledge about the efficacy and safety of vaccines is necessary.

\section{Ethical Approval and Informed Consent}

The study protocol was approved by the research and ethics committee of the Department of Clinical Pharmacology, KUMS under protocol no.71. All participants provided their consent prior to taking part in the study. Participation was completely voluntary, and participants were able to withdraw from the study anytime without any consequence.

\section{Acknowledgment}

The authors would like to sincerely thank Dr. Pakeer Oothuman, former Professor of Medical Parasitology at the Universiti Kebangsaan Malaysia and International Islamic University Malaysia for editing the manuscript.
Faculties and staff of Kabul University of Medical Sciences are also gratefully appreciated for their participation in the study.

\section{Disclosure}

The authors declare no conflicts of interest.

\section{References}

1. Wu Z, McGoogan JM. Characteristics of and important lessons from the coronavirus disease 2019 (COVID-19) outbreak in China: summary of a report of 72314 cases from the Chinese center for disease control and prevention. JAMA. 2020;323(13):1239-1242. doi:10.10 01/jama.2020.2648

2. WHO. COVID-19 Update. Vol. 2021. June, 2021.

3. Jeon M, Kim J, Oh CE, Lee J-Y. Adverse events following immunization associated with coronavirus disease 2019 vaccination reported in the mobile vaccine adverse events reporting system. $J$ Korean Med Sci. 2021;36(17). doi:10.3346/jkms.2021.36.e114

4. Abdulla ZA, Al-Bashir SM, Al-Salih NS, Aldamen AA, Abdulazeez MZ. A summary of the SARS-CoV-2 vaccines and technologies available or under development. Pathogens. 2021;10 (7):788. doi:10.3390/pathogens 10070788

5. Sathian B, Asim M, Banerjee I, et al. Development and implementation of a potential coronavirus disease 2019 (COVID-19) vaccine: a systematic review and meta-analysis of vaccine clinical trials. Nepal J Epidemiol. 2021;11(1):959. doi:10.3126/nje.v11i1.36163

6. Meo S, Bukhari I, Akram J, Meo A, Klonoff D. COVID-19 vaccines: comparison of biological, pharmacological characteristics and adverse effects of Pfizer/BioNTech and moderna vaccines. Eur Rev Med Pharmacol Sci. 2021;25(3):1663-1669.

7. Mascellino MT, Di Timoteo F, De Angelis M, Oliva A. Overview of the main anti-SARS-CoV-2 vaccines: mechanism of action, efficacy and safety. Infect Drug Resist. 2021;14:3459. doi:10.2147/IDR. S315727

8. Kyriakidis NC, López-Cortés A, González EV, Grimaldos AB, Prado EO. SARS-CoV-2 vaccines strategies: a comprehensive review of Phase 3 candidates. Npj Vaccines. 2021;6(1):1-17. doi:10.1038/ s41541-021-00292-w

9. Gee J, Marquez P, Su J. First month of COVID-19 vaccine safety monitoring - United States, December 14, 2020-January 13, 2021. MMWR Morb Mortal Wkly Rep. 2021;70(8):283-288. doi:10.15585/ mmwr.mm7008e3

10. Voysey M, Clemens SAC, Madhi SA, et al. Safety and efficacy of the ChAdOx1 nCoV-19 vaccine (AZD1222) against SARS-CoV-2: an interim analysis of four randomised controlled trials in Brazil, South Africa, and the UK. Lancet. 2021;397(10269):99-111.

11. Hatmal MMM, Al-Hatamleh MA, Olaimat AN, et al. Side effects and perceptions following COVID-19 vaccination in Jordan: a randomized, Cross-Sectional Study implementing machine learning for predicting severity of side effects. Vaccines. 2021;9(6):556. doi:10.3390/vaccines 9060556

12. Shekhar R, Sheikh AB, Upadhyay S, et al. COVID-19 vaccine acceptance among health care workers in the United States. Vaccines. 2021;9(2):119. doi:10.3390/vaccines9020119

13. COVID C. Response TeamFood and Drug Administration. Allergic reactions including anaphylaxis after receipt of the first dose of Pfizer-BioNTech COVID-19 vaccine-United States, December 1423, 2020. MMWR Morb Mortal Wkly Rep. 2021;70(2):46-51. doi:10.15585/mmwr.mm7002e1

14. Carli G, Nichele I, Ruggeri M, Barra S, Tosetto A. Deep vein thrombosis (DVT) occurring shortly after the second dose of mRNA SARS-CoV-2 vaccine. Intern Emerg Med. 2021;16 (3):803-804. doi:10.1007/s11739-021-02685-0 
15. Farooq F, Rathore FA. COVID-19 vaccination and the challenge of infodemic and disinformation. J Korean Med Sci. 2021;36:10. doi:10.3346/jkms.2021.36.e78

16. Control CfD, Prevention. Possible Side Effects After Getting a COVID-19; vaccine; 2021.

17. El-Shitany NA, Harakeh S, Badr-Eldin SM, et al. Minor to moderate side effects of Pfizer-BioNTech COVID-19 vaccine among Saudi residents: a retrospective cross-sectional study. Int $\mathrm{J}$ Gen Med. 2021;14:1389. doi:10.2147/IJGM.S310497

18. Sah R, Shrestha S, Mehta R, et al. AZD1222 (Covishield) vaccination for COVID-19: experiences, challenges and solutions in Nepal. Travel Med Infect Dis. 2021. doi:10.1016/j.tmaid.2021.10 1989
19. Huh K, Kim Y-E, Radnaabaatar M, et al. Estimating baseline incidence of conditions potentially associated with vaccine adverse events: a call for surveillance system using the Korean National Health Insurance Claims Data. J Korean Med Sci. 2021;36(9):9. doi:10.3346/jkms.2021.36.e67

20. Wise J. Covid-19: European Countries Suspend Use of OxfordAstraZeneca Vaccine After Reports of Blood Clots. British Medical Journal Publishing Group; 2021.

21. Menni C, Klaser K, May A, et al. Vaccine side-effects and SARS-CoV-2 infection after vaccination in users of the COVID Symptom Study app in the UK: a prospective observational study. Lancet Infect Dis. 2021;21 (7):939-949. doi:10.1016/S1473-3099(21)00224-3

\section{Publish your work in this journal}

Infection and Drug Resistance is an international, peer-reviewed openaccess journal that focuses on the optimal treatment of infection (bacterial, fungal and viral) and the development and institution of preventive strategies to minimize the development and spread of resistance. The journal is specifically concerned with the epidemiology of antibiotic resistance and the mechanisms of resistance development and diffusion in both hospitals and the community. The manuscript management system is completely online and includes a very quick and fair peerreview system, which is all easy to use. Visit http://www.dovepress.com/ testimonials.php to read real quotes from published authors. 